\title{
Neuralgia, CTCAE
}

National Cancer Institute

\section{Source}

National Cancer Institute. Neuralgia, CT CAE. NCI Thesaurus. Code C143703.

A disorder characterized by intense painful sensation along a nerve or group of nerves. 